\title{
Scattering amplitudes for the rationally extended $P T$ symmetric complex potentials
}

\author{
Nisha Kumari ${ }^{a *}$, Rajesh Kumar Yadav ${ }^{b \dagger}$, Avinash Khare ${ }^{c \ddagger}$ Bijan Bagchi ${ }^{d \S}$ and \\ Bhabani Prasad Mandal ${ }^{a}$
}

September 22, 2018

${ }^{a}$ Department of Physics, Banaras Hindu University, Varanasi-221005, India.

${ }^{b}$ Department of Physics, S. P. College, S. K. M. University, Dumka-814101, India.

${ }^{c}$ Department of Physics, Savitribai Phule Pune University, Pune-411007, India.

${ }^{d}$ Department of Physics, School of Natural Science, Shiv Nadar University, Greater Noida, UP-201314 India.

\begin{abstract}
In this paper, we consider the rational extensions of two different $P T$ symmetric complex potentials namely the asymptotically vanishing Scarf II and asymptotically non-vanishing Rosen-Morse II [ RM-II] potentials and obtain bound state eigenfunctions in terms of newly found exceptional $X_{m}$ Jacobi polynomials and also some new type of orthogonal polynomials respectively. By considering the asymptotic behaviour of the exceptional polynomials, we obtain the reflection and transmission amplitudes for them and discuss the various novel properties of the corresponding amplitudes.
\end{abstract}

\section{Introduction}

Recent discovery of exceptional orthogonal polynomials (EOPs) (also known as $X_{m}$ Laguerre and $X_{m}$ Jacobi orthogonal polynomials with $\left.m=0,1,2, \ldots,.\right)$ [1, 2, 3, 4], has motivated researchers to search for new exactly solvable potentials whose bound state eigenfunctions are in terms of these EOPs. Interestingly it has been observed that the newly discovered potentials are the rational extension of the corresponding conventional

*e-mail address: nishaism0086@gmail.com (N.K)

${ }^{\dagger}$ e-mail address: rajeshastrophysics@gmail.com (R.K.Y)

‡e-mail: khare@physics.unipune.ac.in (A.K)

$\S$ e-mail address:bbagchi123@gmail.com (B.B)

ฯe-mail address: bhabani.mandal@gmail.com (B.P.M). 
potentials [5, 6, 7, 8]. These potentials are rationally extended trigonometric Scarf (ScarfI), generalized Pöschl Teller (GPT) and radial oscillator potentials. Remarkably, these potentials can be cast in the framework of supersymmetric quantum mechanics (SQM) and they have been shown to satisfy the translational shape invariance (SI) condition [9].

In the last few years, researchers have also discovered another category of rationally extended potentials whose bound state eigenfunctions are not in terms of the EOPs but rather they are written in terms of some new polynomials (which in turn can be expressed in terms of the classical orthogonal polynomials). These rationally extended potentials do not satisfy the usual translational SI property, but instead they satisfy an unfamiliar type of extended SI property. The potentials belong to this category are rationally extended Rosen-Morse II, Eckart etc [10, 11]. It is important here to note that the bound state eigenvalues of all these rationally extended potentials are the same as those of their conventional counterparts i.e. they are iso-spectral.

Recently, fully consistent quantum theories have been developed for certain class of non-hermitian systems. The parity $(\mathrm{P})$ and time reversal $(\mathrm{T})$, two important discrete symmetries play extremely important role in such formulations. It has been shown that the PT symmetric (combined parity and time reversal symmetry) non-hermitian systems can have the spectrum real if the PT symmetric is unbroken [12] and consequently a fully consistent quantum theory with unitary time evolution is constructed in a modified Hilbert space [13]. Since the last one and half decades, the PT symmetric non-hermitian systems have been developed considerably and have found several applications in optics as well as other branches of physics [14, 15, 16, 17, 18, 19, 20, 21]. Some of the important properties such as the existence of spectral singularity (divergence of both reflection and transmission co-efficients at a point) [22, 23, 24, 25, 26], exceptional points (coalesce of two energy levels along with their eigenfunctions at certain points in the complex plane) [27, 28, 29, 30], Handedness effect (dependence of reflection coefficient on the direction of wave) [31] have been also observed in such systems.

In the last few years while much work has been done on the PT-symmetric potentials, we find that comparatively not enough work has been done about the rationally extended PT symmetric (REPTS) complex potentials. However, considerable work has already been done about the rationally extended real (Hermitian) potentials and bound state eigenstates as well as scattering matrix has already been reported for them [34, 35, 36, 37. For example, while rational extension of real (Hermitian) Rosen-Morse II potential has been extensively discussed [10, it seems there has been no discussion about the corresponding rationally extended, complex PT invariant Rosen-Morse II potential. Further, to the best of our knowledge, only one REPTS complex potential corresponding to the conventional PT symmetric complex Scarf II potential [32] has so far been discussed in the literature. In particular, it has been shown that the bound state eigenfunctions of rationally extended PT-symmetric complex Scarf II potential are in terms of $X_{m}$ Jacobi EOPs [33]. However, as far as we know, the corresponding reflection and transmission amplitudes $r(k)$ and $t(k)$ respectively are not known in the literature.

The purpose of this paper is to fill the missing gap and discuss bound state eigenstates 
as well as reflection and transmission coefficients of few rationally extended PT-symmetric complex potentials. In particular, in this paper, we obtain bound state eigenfunctions as well as $r(k)$ and $t(k)$ for the rationally extended $P T$ symmetric complex Rosen-Morse II potential. Further, we construct one more complex and $P T$ symmetric rationally extended Rosen-Morse II potential and obtain its bound state eigenfunctions. Further, we obtain $r(k)$ and $t(k)$ for both the forms of complex PT-symmetric rationally extended RosenMorse II potentials. Recently an important symmetry i.e., the parametric symmetry in the PT symmetric conventional as well as rationally extended Scarf II potentials has been observed. Under this symmetry while the PT symmetric Scarf II potential remains invariant (and has two different sets of bound states), however the rationally extended $P T$ symmetric complex Scarf II potential [38] is not invariant and one gets another rationally extended PT symmetric complex Scarf II potential. It is thus of interest to inquire how $r(k)$ and $t(k)$ change under the parametric symmetry.

The organization of this paper is as follows:

In section 2, we briefly discuss the bound state eigenfunctions of the rationally extended PT symmetric complex Scarf II and Rosen-Morse II potentials. The parametric symmetry in the case of the extended PT symmetric complex Scarf II is also discussed. The scattering amplitude, i.e. $r(k)$ and $t(k)$ for these potentials is obtained in section 3 where some interesting properties of $r(k)$ and $t(k)$ are highlighted. Finally we summarize the work done in this paper in section 4.

\section{Bound states: REPTS potentials}

In this section, we briefly discuss the bound state eigenfunctions of the two rationally extended complex PT symmetric potentials .

\subsection{REPTS complex Scarf-II potential}

The well known complex and PT symmetric conventional Scarf II potential [39] which is translationally $\mathrm{SI}$ is given by

$$
V(x, A, B)=\left(-B^{2}-A(A+1)\right) \operatorname{sech}^{2} x+i B(2 A+1) \operatorname{sech} x \tanh x ; \quad A>0 .
$$

The bound states energy eigenvalues and the eigenfunctions are known to be 33

$$
E_{n}^{(A)}=-(A-n)^{2} ; \quad n=0,1,2 \ldots n_{\max }<A,
$$

and

$$
\psi_{n}^{(A, B)}(x)=N_{n}^{(\alpha, \beta)}(\operatorname{sech} x)^{A} \exp \left(-i B \tan ^{-1}(\sinh x)\right) P_{n}^{(\alpha, \beta)}(i \sinh x) ; \quad-\infty<x<\infty,
$$

where $P_{n}^{(\alpha, \beta)}(z)$ is classical Jacobi polynomial, $N_{n}^{(\alpha, \beta)}$ is the normalization constant and the parameters $\alpha=B-A-\frac{1}{2}$ and $\beta=-B-A-\frac{1}{2}$. An interesting symmetry of 
this conventional PT symmetric complex Scarf II potential has been observed under a parametric transformation [40] i.e. $B \longleftrightarrow\left(A+\frac{1}{2}\right)$. Under this transformation the potential given in Eq. (11) remains invariant. This symmetry implies another set of bound state solutions with the eigenstates given by

$$
E_{n}^{(B)}=-\left(B-n-\frac{1}{2}\right)^{2} ; \quad n=0,1,2, \ldots, n_{\max }<B-\frac{1}{2},
$$

and

$$
\psi_{n}^{\left(B \leftrightarrow A+\frac{1}{2}\right)}(x)=N_{n}^{(\gamma, \delta)}(\operatorname{sech} x)^{B-\frac{1}{2}} \exp \left(-i\left(A+\frac{1}{2}\right) \tan ^{-1}(\sinh x)\right) P_{n}^{(\gamma, \delta)}(i \sinh x),
$$

where the new parameters $\gamma=-\alpha=A-B+\frac{1}{2}$ and $\delta=\beta=-A-B-\frac{1}{2}$.

At this point we observe that the potential given by Eq. (1) has been extended rationally [33] for any positive integer values of $m$, i.e.

$$
\begin{aligned}
V_{m}(x, A, B) & =V(x, A, B)+2 m(2 B-m+1)+(2 B-m+1) \\
& \times[(-2 A-1)+(2 B+1) i \sinh x] \frac{P_{m-1}^{(-\alpha, \beta)}(i \sinh x)}{P_{m}^{(-\alpha-1, \beta-1)}(i \sinh x)} \\
& -\frac{(2 B-m+1)^{2} \cosh ^{2} x}{2}\left(\frac{P_{m-1}^{(-\alpha, \beta)}(i \sinh x)}{P_{m}^{(-\alpha-1, \beta-1)}(i \sinh x)}\right)^{2}
\end{aligned}
$$

where $V(x, A, B)$ is the conventional $P T$ symmetric complex Scarf II potential given in Eq. (11). Like the complex $P T$ symmetric Scarf II potential given by Eq. (1), these extended potentials are also SI under the translation of the parameters $A \rightarrow A-1$ and the energy eigenvalues are (real) and the same (i.e. isospectral) as that of the conventional one as given by (2). The bound state eigenfunctions corresponding to the extended potential (6) are given by

$$
\psi_{n, m}^{(A, B)}(x)=N_{n, m}^{(\alpha, \beta)} \times \frac{\left(1+\sinh ^{2} x\right)^{-\frac{A}{2}} \exp \left\{-i B \tan ^{-1}(\sinh x)\right\}}{P_{m}^{(-\alpha-1, \beta-1)}(i \sinh x)} \hat{P}_{n+m}^{(\alpha, \beta)}(i \sinh x),
$$

where $N_{n, m}^{(\alpha, \beta)}$ is a normalization constant and $\hat{P}_{n+m}^{(\alpha, \beta)}(i \sinh x)$ is $X_{m}$ Jacobi EOPs. orthogonal polynomials. As expected, for $m=0$, the above rationally extended potential (as well as the corresponding eigenfunctions) reduce to the conventional one as given by Eqs. (11) and (2) while for $m=1$ the potential corresponds to the case of $X_{1}$ EOPs [32]. Here it is important to note that unlike the conventional potential Eq. (11), the rationally extended potential Eq. (6) is not invariant under the transformation $B \longleftrightarrow A+\frac{1}{2}$, rather it goes 
over to another extended potential given by

$$
\begin{aligned}
V_{m}\left(x, B \leftrightarrow A+\frac{1}{2}\right) & =V(x)+2 m(2 A-m+2)+(2 A-m+2) \\
& \times[(-2 B)+(2 A+2) i \sinh x] \frac{P_{m-1}^{(-\gamma, \delta)}(i \sinh x)}{P_{m}^{(-\gamma-1, \delta-1)}(i \sinh x)} \\
& -\frac{(2 A-m+2)^{2} \cosh ^{2} x}{2}\left(\frac{P_{m-1}^{(-\gamma, \delta)}(i \sinh x)}{P_{m}^{(-\gamma-1, \delta-1)}(i \sinh x)}\right)^{2} .
\end{aligned}
$$

The energy eigenvalues of this potential are isospectral to that of the conventional potential obtained after the transformation $B \longleftrightarrow A+\frac{1}{2}$ and are given by Eq. (41). The bound state eigenfunctons corresponding to this new rationally extended potential are given by

$$
\psi_{(n, m)}^{\left(B \leftrightarrow A+\frac{1}{2}\right)}(x)=N_{(n, m)}^{(\gamma, \delta)} \times \frac{(\operatorname{sech} x)^{A} \exp \left(-i B \tan ^{-1}(\sinh x)\right)}{P_{m}^{(-\gamma-1, \delta-1)}(i \sinh x)} \hat{P}_{n+m}^{(\gamma, \delta)}(i \sinh x) .
$$

The above rationally extended potential is also SI under the translation of parameter $B \longrightarrow B-1$.

\section{$2.2 \quad$ REPTS complex RM-II potential}

This potential belongs to the second category of the rationally extended potentials whose bound state solutions are not in the exact form of EOPs, they are in the form of some types of new polynomials [10]. These polynomials can be written in terms of classical Jacobi polynomials. Further, these potentials do not satisfy the usual SI property, but instead they satisfy an unusual type of enlarge SI property where both the potential parameters as well as the order of the polynomials change.

Using first-order SUSY QM, Quesne [10] obtained the rationally extended real RosenMorse II (RM-II) potential by considering the conventional real Rosen-Morse II potential. We can then obtain the rationally extended PT symmetric complex RM-II potentials by essentially following the approach of [10] by considering the PT symmetric complex RMII potential [41] (which can be obtained by changing the potential parameter $B \longrightarrow i B$ in the conventional real potential) as

$$
V_{A, i B}(x)=-A(A+1) \operatorname{sech}^{2} x+2 i B \tanh x, \quad-\infty<x<\infty,
$$

where $A>0$. The bound state energy eigenvalues turn out to be real

$$
E_{n}^{(A, i B)}=-(A-n)^{2}+\frac{B^{2}}{(A-n)^{2}}, \quad n=0,1,2 \ldots, n_{\max }<A .
$$

The corresponding wavefunctions in terms of classical Jacobi polynomials $P^{\left(A-n+\frac{i B}{A-n}, A-n-\frac{i B}{A-n}\right)}(z)$ are

$$
\psi_{n}^{(A, i B)}(x) \propto(1-\tanh x)^{\frac{1}{2}\left(A-n+\frac{i B}{A-n}\right)}(1+\tanh x)^{\frac{1}{2}\left(A-n-\frac{i B}{A-n}\right)} P_{n}^{\left(A-n+\frac{i B}{A-n}, A-n-\frac{i B}{A-n}\right)}(z),
$$


where $z=\tanh x$. Similar to the real case [10], this complex PT symmetric potential can be extended rationally by determining all possible polynomials-type, nodeless solutions $\phi(x)$ of the Schrödinger equation

$$
-\frac{d^{2} \phi(x)}{d x^{2}}+V_{A, i B}(x) \phi(x)=E \phi(x)
$$

with the factorization energy $E<E_{0}^{(A, i B)}=-A^{2}+\frac{B^{2}}{A^{2}}$. Out of all the possible solutions of $\phi(x)$, two independent polynomial type solutions $\phi_{1}(x)$ and $\phi_{2}(x)$ with the energy $E_{1}$ and $E_{2}$ respectively have been constructed (for detail see Ref. [10]). On putting some restrictions on the parameters $A$ and $B$, three acceptable polynomial-type, nodeless solutions are obtained. By considering the conventional potential with some different $A^{\prime}$ i.e.

$$
V^{(+)}(x)=V_{\left(A^{\prime}, i B\right)}(x)
$$

the rationally-extended $P T$ symmetric Rosen Morse-II potential $V^{(-)}(x)$ with given $A$ and $B$ is obtained as

$$
V^{(-)}(x)=V_{(A, i B, e x t)}(x)=V_{(A, i B)}(x)+V_{(A, i B, \text { rat })}(x),
$$

where

$$
V_{(A, i B, r a t)}(x)=2\left(1-z^{2}\right)\left\{2 z \frac{\dot{g}_{m}^{(A, i B)}}{g_{m}^{(A, i B)}}-\left(1-z^{2}\right)\left[\frac{\ddot{g}_{m}^{(A, i B)}}{g_{m}^{(A, i B)}}-\left(\frac{\dot{g}_{m}^{(A, i B)}}{g_{m}^{(A, i B)}}\right)^{2}\right]-m\right\},
$$

with $z=\tanh x$. Here dot denotes a derivative with respect to $z$.

According to the different conditions on the potential parameters they categorize the extended potentials into three types. Out of these three, first two are isospectral to their conventional one. Both of these extended potentials are equivalent, they differ only in the range of the potential parameters.

Here we consider type I case and define the parameter $A^{\prime}=A+1$, and the other parameters as

$$
\begin{aligned}
g_{m}^{(A, i B)}(z) & =P_{m}^{\left(\alpha_{m}, \beta_{m}\right)}(z) \\
\text { where } \quad \alpha_{m} & =A+1-m+\frac{i B}{A+1-m}, \\
\beta_{m} & =A+1-m-\frac{i B}{A+1-m} ; \quad m=1,2,3, \ldots ; \quad A>m-1,
\end{aligned}
$$

one obtains the rationally extended RM-II potentials, $V^{(-)}(x)=V_{(A, i B, e x t)}(x)$ isospectral to the potentials $V^{(+)}(x)$ with a bound state spectrum

$$
E_{n}=-(A+1-n)^{2}+\frac{B^{2}}{(A+1-n)^{2}}, \quad n=0,1,2 \ldots n_{\max }, \quad n_{\max }<A+1 .
$$


The corresponding bound state eigenfunctions of $V^{(+)}(x)$ and $V^{(-)}(x)$ are given by

$$
\psi_{n}^{(+)}(x) \propto(1-z)^{\frac{\alpha_{n}}{2}}(1+z)^{\frac{\beta_{n}}{2}} P_{n}^{\left(\alpha_{n}, \beta_{n}\right)}(z)
$$

and

$$
\psi_{n}^{(-)}(x) \propto \hat{A} \psi_{n}^{(+)}(x)
$$

respectively. Where the parameters $\alpha_{n}=A+1-n+\frac{i B}{A+1-n}, \quad \beta_{n}=A+1-n-\frac{i B}{A+1-n}$ and operator

$$
\hat{A}=\left(1-z^{2}\right) \frac{d}{d z}+\frac{i B}{A+1}+(A+1) z-\frac{2\left(m+\alpha_{m}\right)\left(m+\beta_{m}\right)}{2 m+\alpha_{m}+\beta_{m}} \frac{g_{m-1}^{(A-1, i B)}}{g_{m}^{(A, i B)}} .
$$

Using $\hat{A}$ and $z$ in Eq. (20) and after simple calculation the wavefunction $\psi_{n}^{(-)}(x)$ is given by

$$
\psi_{n}^{(-)}(x) \propto \frac{(1-z)^{\frac{\alpha_{n}}{2}}(1+z)^{\frac{\beta_{n}}{2}}}{g_{m}^{(A, i B)}(z)} y_{\nu}^{(A, i B)}(z), \quad \nu=m+n-1
$$

where $y_{\nu}^{(A, i B)}(z)$ is some new type of $\nu$ th-degree polynomial in $z$ defined in terms of classical Jacobi polynomials as

$$
\begin{aligned}
y_{\nu}^{(A, i B)}(z)= & \frac{2\left(n+\alpha_{n}\right)\left(n+\beta_{n}\right)}{2 n+\alpha_{n}+\beta_{n}} g_{m}^{(A, i B)}(z) P_{n-1}^{\left(\alpha_{n}, \beta_{n}\right)}(z)- \\
& \frac{2\left(m+\alpha_{m}\right)\left(m+\beta_{m}\right)}{2 m+\alpha_{m}+\beta_{m}} g_{m-1}^{(A-1, i B)}(z) P_{n}^{\left(\alpha_{n}, \beta_{n}\right)}(z) .
\end{aligned}
$$

The superpotential corresponding to this potential is given by

$$
\begin{aligned}
\bar{W}(x) & =-\log \left(\psi_{0}^{(-)}(x)\right)^{\prime} \\
& =\frac{i B}{A+1}+(A+1) z-\left(1-z^{2}\right)\left(\frac{\dot{g}_{m-1}^{(A-1, i B)}}{g_{m-1}^{(A-1, i B)}}-\frac{\dot{g}_{m}^{(A, i B)}}{g_{m}^{(A, i B)}}\right) .
\end{aligned}
$$

The partner potentials are

$$
\bar{V}^{( \pm)}(x)=\bar{W}^{2}(x) \pm \bar{W}(x)
$$

where

$$
\begin{aligned}
\bar{V}^{(+)}(x) & =V^{(-)}(x)=V_{(A, i B, e x t)}^{(m)}(x) \\
\text { and } \quad \bar{V}^{(-)}(x) & =V_{(A-1, i B, e x t)}^{(m-1)}(x) .
\end{aligned}
$$

Similar to the real extended case [10], we see that the above rationally extended complex $P T$ symmetric potential also satisfies an unfamiliar type of extended SI conditions where the partner potential $\bar{V}^{(-)}(x)$ is obtained by translating the potential parameter $A \rightarrow A-1$ (as in the usual SI condition) and the degree of the polynomial $m \rightarrow m-1$. 


\section{$3 \quad$ Scattering amplitudes: REPTS complex poten- tials}

In the case of one dimensional systems, the transmission and reflection amplitudes can be calculated easily by assuming the asymptotic behavior of the wavefunctions at $x \longrightarrow \pm \infty$.

In this section, we first consider an asymptotically vanishing potential, the rationally extended complex Scarf II potential and then a non-vanishing potential, the rationally extended complex RM-II potential and obtain the scattering amplitudes in both the cases.

\subsection{REPTS complex Scarf II potential}

For simplicity, we first consider the potential whose bound state eigenfunctions are associated with the $X_{1}$ Jacobi polynomial and then we generalize it to the $X_{m}$ case. For $m=1$, the bound state eigenfunctions of the rationally extended complex PT symmetric Scarf II potential are given by Eq. (7), i.e.

$$
\psi_{n, 1}(x) \propto \frac{\left(1+\sinh ^{2} x\right)^{-\frac{A}{2}} \exp \left\{-i B \tan ^{-1}(\sinh x)\right\}}{P_{1}^{(-\alpha-1, \beta-1)}(i \sinh x)} \hat{P}_{n+1}^{(\alpha, \beta)}(i \sinh x),
$$

where $\hat{P}_{n+1}^{(\alpha, \beta)}(i \sinh x)$ is the $X_{1}$ Jacobi polynomial [1, 2] written in terms of classical Jacobi polynomial as

$$
\begin{aligned}
\hat{P}_{n+1}^{(\alpha, \beta)}(i \sinh x) & =\frac{1}{2(\alpha+\beta+2 n)}\left[\{(b-i \sinh x)(\alpha+\beta+n)+2 b\} P_{n}^{(\alpha, \beta)}(i \sinh x)\right. \\
& \left.-2 P_{n-1}^{(\alpha, \beta)}(i \sinh x)\right]
\end{aligned}
$$

with $b=\frac{\beta+\alpha}{\beta-\alpha}$.

Using the properties of Jacobi polynomials 42

$2 P_{n-1}^{(\alpha, \beta)}(z)=\frac{(2 n+\alpha+\beta)\left(1-z^{2}\right)}{(n+\alpha)(n+\beta)} \frac{d}{d z} P_{n}^{(\alpha, \beta)}(z)-\frac{n[(\alpha-\beta)-(2 n+\alpha+\beta) z]}{(n+\alpha)(n+\beta)} P_{n}^{(\alpha, \beta)}(z)$,

the $X_{1}$ Jacobi polynomial as given by Eq. (28) can be simplified in terms of $P_{n}^{(\alpha, \beta)}(i \sinh x)$

$$
\begin{aligned}
\hat{P}_{n+1}^{(\alpha, \beta)}(i \sinh x) & =\frac{1}{2(\alpha+\beta+2 n)}[\{((b-i \sinh x)(\alpha+\beta+n)+2 b) \\
& \left.+\frac{n[(\alpha-\beta)-(2 n+\alpha+\beta) i \sinh x]}{(n+\alpha)(n+\beta)}\right\} P_{n}^{(\alpha, \beta)}(i \sinh x) \\
& \left.-\frac{(2 n+\alpha+\beta)}{(n+\alpha)(n+\beta)}\left(\frac{\cosh x}{i}\right) \frac{d}{d x} P_{n}^{(\alpha, \beta)}(i \sinh x)\right] .
\end{aligned}
$$


In terms of hypergeometric function, the classical Jacobi Polynomial $P_{n}^{(\alpha, \beta)}(i \sinh x)$ can be written as

$$
P_{n}^{(\alpha, \beta)}(i \sinh x)=(-1)^{n} \frac{\Gamma(n+\beta+1)}{n ! \Gamma(1+\beta)} F\left(n+\alpha+\beta+1,-n ; 1+\beta ; \frac{1+i \sinh x}{2}\right) .
$$

Using Eqs. (30), (31) and (27), we get the bound state eigenfunctions in terms of hypergeometric functions.

To get the scattering state solutions, we must retain the second solution which has been discarded in the case of bound states since it diverged asymptotically. The second solution is included by replacing the hypergeometric function $F(a, b, c, \tilde{z})$ by

$$
F(a, b ; c ; \tilde{z})=C_{1} F(a, b ; c ; \tilde{z})+C_{2} \tilde{z}^{1-c} F(a-c+1, b-c+1 ; 2-c ; \tilde{z}),
$$

where $C_{1}$ and $C_{2}$ are two constants. Further, instead of the parameter $n$ labeling the number of nodes, one must use the wavenumber $k$ so that one gets the asymptotic wavefunctions in terms of $e^{ \pm i k x}$ as $x \rightarrow \pm \infty$.

To get the asymptotic wavefunctions at $x \rightarrow \pm \infty$ now we use another properties of Hypergeometric function 42

$$
\begin{aligned}
F(a, b ; c ; \tilde{z})= & \frac{\Gamma(c) \Gamma(b-a)}{\Gamma(b) \Gamma(c-a)}(-1)^{a} \tilde{z}^{-a} F\left(a, a+1-c ; a+1-b ; \frac{1}{\tilde{z}}\right)+ \\
& \frac{\Gamma(c) \Gamma(a-b)}{\Gamma(a) \Gamma(c-b)}(-1)^{b} \tilde{z}^{-b} F\left(b, b+1-c ; b+1-a ; \frac{1}{\tilde{z}}\right)
\end{aligned}
$$

Now using Eq.(33) and then taking the asymptotic behavior of the Eq. (27) and after replacing $n$ by $A+i k$, we get

$$
\begin{aligned}
\lim _{x \rightarrow \infty} \psi(k, x) & \simeq \frac{\exp \left(-\frac{i \pi B}{2}\right)}{(-i B)}\left[\left(C_{1} a_{k}+C_{2} c_{k}(-1)^{(-\beta)}\right)\left(\frac{1}{4 i}\right)^{(A-i k)} \xi_{1}(k) e^{-i k x}\right. \\
& \left.+\left(C_{1} b_{k}+C_{2} d_{k}(-1)^{(-\beta)}\right)\left(\frac{1}{4 i}\right)^{(A+i k)} e^{i k x}\right] .
\end{aligned}
$$

and

$$
\begin{aligned}
\lim _{x \rightarrow-\infty} \psi(k, x) & \simeq \frac{\exp \left(\frac{i \pi B}{2}\right)}{(-i B)}\left[\left(C_{1} a_{k}(-1)^{A-i k}+C_{2} c_{k}(-1)^{-\left(B+i k+\frac{1}{2}\right)}\right)\left(\frac{1}{4 i}\right)^{A-i k}\right. \\
& \times \xi_{1}(k) e^{i k x}+\left(C_{1} b_{k}(-1)^{A+i k}+C_{2} d_{k}(-1)^{\left(-B+i k-\frac{1}{2}\right)}\right) \\
& \left.\times\left(\frac{1}{4 i}\right)^{A+i k} e^{-i k x}\right] .
\end{aligned}
$$

where

$a_{k}=\frac{\Gamma(1+\beta) \Gamma(-2 A-2 i k-\alpha-\beta-1)}{\Gamma(-A-i k) \Gamma(-A-i k-\alpha)}, \quad b_{k}=\frac{\Gamma(1+\beta) \Gamma(2 A+2 i k+\alpha+\beta+1)}{\Gamma(A+i k+\alpha+\beta+1) \Gamma(A+i k+\beta+1)}$, 
$c_{k}=\frac{\Gamma(1-\beta) \Gamma(-2 A-2 i k-\alpha-\beta-1)}{\Gamma(-A-i k-\beta) \Gamma(-A-i k-\alpha-\beta)}, \quad d_{k}=\frac{\Gamma(1-\beta) \Gamma(2 A+2 i k+\alpha+\beta+1)}{\Gamma(A+i k+\alpha+1) \Gamma(1+A+i k)}$

and

$$
\xi_{1}(k)=1+\frac{(2 i k)}{(A+i k+\alpha)(A+i k+\beta)} .
$$

The asymptotic behavior of the wave functions due to left incident wave is given by

$$
\begin{aligned}
\lim _{x \rightarrow-\infty} \psi_{\nu}(x) & \simeq e^{i k x}+r_{\text {left }}(k) e^{-i k x} \\
\text { and } \quad \lim _{x \rightarrow \infty} \psi_{\nu}(x) & \simeq t_{\text {left }}(k) e^{i k x}
\end{aligned}
$$

On comparing Eqs. (34) and (36), one can easily obtain the constants $C_{1}$ and $C_{2}$

$$
C_{1}=\frac{\exp \left(-\frac{i \pi B}{2}\right)\left(\frac{1}{4 i}\right)^{(-A+i k)}(-1)^{\left(B+i k+\frac{1}{2}\right)}}{a_{k}\left\{(-1)^{-\beta}-(-1)^{\beta}\right\}} \times \frac{1}{\xi_{1}(k)}
$$

and

$$
C_{2}=\frac{-\exp \left(\frac{-i \pi B}{2}\right)\left(\frac{1}{4 i}\right)^{(-A+i k)}(-1)^{(-A+i k)}}{c_{k}\left\{(-1)^{-\beta}-(-1)^{\beta}\right\}} \times \frac{1}{\xi_{1}(k)} .
$$

In this way, we obtain the left transmission and reflection amplitudes

$$
t_{\text {left }}(k)=t_{\text {left }}^{\text {usual }}(k) \times\left(\frac{\left(i k-\frac{1}{2}\right)^{2}-B^{2}}{\left(i k+\frac{1}{2}\right)^{2}-B^{2}}\right)
$$

and

$$
r_{\text {left }}(k)=r_{\text {left }}^{\text {usual }}(k)\left(\frac{\left(i k-\frac{1}{2}\right)^{2}-B^{2}}{\left(i k+\frac{1}{2}\right)^{2}-B^{2}}\right),
$$

where $t_{\text {left }}^{\text {usual }}(k)$ and $r_{\text {left }}^{\text {usual }}(k)$ are the transmission and reflection amplitudes for the usual $P T$ symmetric complex Scarf II potential [43] and are given by

$$
t_{\text {left }}^{u s u a l}(k)=\frac{\Gamma(-A-i k) \Gamma(1+A-i k) \Gamma\left(\frac{1}{2}-B-i k\right) \Gamma\left(\frac{1}{2}+B-i k\right)}{\Gamma(-i k) \Gamma(1+i k) \Gamma^{2}\left(\frac{1}{2}-i k\right)},
$$

and

$$
r_{\text {left }}^{\text {usual }}(k)=t_{\text {left }}^{\text {usual }}(k) \times i\left[\frac{\cos \pi A \sin \pi B}{\cosh \pi k}+\frac{\sin \pi A \cos \pi B}{\sinh \pi k}\right]
$$

respectively. Similar to the left incident wave, for the right incident case the asymptotic wavefunctions are

$$
\begin{aligned}
\lim _{x \rightarrow \infty} \psi_{\nu}(x) & \simeq e^{-i k x}+r_{\text {right }}(k) e^{i k x} \\
\text { and } \quad \lim _{x \rightarrow-\infty} \psi_{\nu}(x) & \simeq t_{\text {right }}(k) e^{-i k x}
\end{aligned}
$$


By following similar procedure, for the case of the right incident wave, using Eqs. (34) and (42) we obtain

$$
t_{\text {right }}(k)=t_{\text {left }}(k)
$$

and

$$
r_{\text {right }}(k)=r_{\text {right }}^{\text {usual }}(k) \times\left(\frac{\left(i k-\frac{1}{2}\right)^{2}-B^{2}}{\left(i k+\frac{1}{2}\right)^{2}-B^{2}}\right)
$$

with

$$
r_{\text {right }}^{\text {usual }}(k)=t_{\text {right }}^{\text {usual }}(k) \times i\left[-\frac{\cos (\pi A) \sin (\pi B)}{\cosh (\pi k)}+\frac{\cos (\pi B) \sin (\pi A)}{\sinh (\pi k)}\right] .
$$

\section{Generalization to the $X_{m}$ case:}

Generalization to the case of $X_{m}$ is straightforward. We start from Eq. (7) where the $n+m$ th degree $X_{m}$ Jacobi orthogonal polynomial $\hat{P}_{n+m}^{(\alpha, \beta)}(i \sinh x)$ can be expressed in terms of the classical Jacobi polynomials by

$$
\begin{aligned}
\hat{P}_{n+m}^{(\alpha, \beta)}(i \sinh x) & =\left\{P_{m}^{(-\alpha-2, \beta)}(i \sinh x)+\frac{2 n(m-\alpha+\beta-1) P_{m-1}^{(-\alpha, \beta)}(i \sinh x)}{(2 m-\alpha+\beta-2)(2 n+\alpha+\beta)}\right. \\
& \left.-\frac{n(\beta+m-1) P_{m-2}^{(-\alpha, \beta)}(i \sinh x)}{(\alpha+n-m+1)(2 m-\alpha+\beta-2)}\right\} P_{n}^{(\alpha, \beta)}(i \sinh x) \\
& +\frac{(m-\alpha+\beta-1)(\alpha+n)}{(\alpha+n-m+1)(2 n+\alpha+\beta)} P_{m-1}^{(-\alpha, \beta)}(i \sinh x) P_{n-1}^{(\alpha, \beta)}(i \sinh x) .
\end{aligned}
$$

On using Eq. (29) in the above equation and by considering the normalization of the Jacobi polynomials

$$
P_{m}^{(\alpha, \beta)}(i \sinh x)=\frac{\Gamma(\alpha+\beta+2 m+1)}{m ! \Gamma(\alpha+\beta+m+1)}\left(\frac{i \sinh x-1}{2}\right)^{m}+(\text { lower degree terms })
$$

and following the same steps as in the $X_{1}$ case it is straightforward to obtain the scattering amplitudes $t_{\text {left }}(k, m)$ and $r_{\text {left }}(k, m)$ in the $X_{m}$ case. We obtain

$$
t_{\text {left }}(k, m)=t_{\text {left }}^{\text {usual }}(k) \times\left(\frac{\left[B^{2}-\left(i k-\frac{1}{2}\right)^{2}\right]+\left(B-i k+\frac{1}{2}\right)(1-m)}{\left[B^{2}-\left(i k+\frac{1}{2}\right)^{2}\right]+\left(B+i k+\frac{1}{2}\right)(1-m)}\right)
$$

and

$$
r_{\text {left }}(k, m)=r_{\text {left }}^{\text {usual }}(k) \times\left(\frac{\left[B^{2}-\left(i k-\frac{1}{2}\right)^{2}\right]+\left(B-i k+\frac{1}{2}\right)(1-m)}{\left[B^{2}-\left(i k+\frac{1}{2}\right)^{2}\right]+\left(B+i k+\frac{1}{2}\right)(1-m)}\right) .
$$

Similarly, $t_{\text {right }}(k, m)$ and $r_{\text {right }}(k, m)$ for the $X_{m}$ case are given by

$$
t_{\text {right }}(k, m)=t_{\text {left }}(k, m)
$$


and

$$
r_{\text {right }}(k, m)=r_{\text {right }}^{\text {usual }}(k) \times\left(\frac{\left[B^{2}-\left(i k-\frac{1}{2}\right)^{2}\right]+\left(B-i k+\frac{1}{2}\right)(1-m)}{\left[B^{2}-\left(i k+\frac{1}{2}\right)^{2}\right]+\left(B+i k+\frac{1}{2}\right)(1-m)}\right) .
$$

As mentioned above, under the parametric symmetry $B \leftrightarrow A+\frac{1}{2}$, while the conventional complex Scarf II potential (1) (and hence the corresponding scattering amplitudes) are invariant, but the rationally extended complex Scarf II potential (6) is not invariant under this parametric transformation but instead we get another extended complex Scarf II potential Eq. (8) . The scattering amplitudes of the new extended potential Eq. (8) can be easily obtained by considering the asymptotic behavior of the bound state solutions given in Eq. (9) or by simply replacing the parameters $B \leftrightarrow A+\frac{1}{2}$ in Eqs. (47) and (48).

\section{Remarks:}

- It is interesting to note that as we go from the conventional to the rationally extended $X_{m}$ case, we get the new expressions for transmission and reflection amplitudes with an extra $m$ dependent term $\left(\frac{\left[B^{2}-\left(i k-\frac{1}{2}\right)^{2}\right]+\left(B-i k+\frac{1}{2}\right)(1-m)}{\left[B^{2}-\left(i k+\frac{1}{2}\right)^{2}\right]+\left(B+i k+\frac{1}{2}\right)(1-m)}\right)$ multiplied with the transmission and reflection amplitudes of the conventional PT invariant potential.

- We can check easily that for $m=0$ the above results correspond to the results of the conventional PT symmetric Scarf II potential [43] and for $m=1$ the results reduce to the results of $X_{1}$ case as given by Eqs. (40) and (43).

- As in the conventional case [43, even in the rationally extended complex Scarf II case the reflection coefficient exhibits the handedness effect i.e., $r_{\text {left }}(k) \neq r_{\text {right }}(k)$.

- Since $\left|\frac{\left[B^{2}-\left(i k-\frac{1}{2}\right)^{2}\right]+\left(B-i k+\frac{1}{2}\right)(1-m)}{\left[B^{2}-\left(i k+\frac{1}{2}\right)^{2}\right]+\left(B+i k+\frac{1}{2}\right)(1-m)}\right|=1$, hence $T(k)$ and $R(k)$ satisfy the same reciprocity, unitarity and other scattering properties as satisfied by the convention $P T$ symmetric Scarf II potential [31, 44].

\subsection{REPTS complex RM-II potential}

This is an asymptotically nonvanishing potential. For such potentials we define the wave numbers $k$ for $x<0$ and $k^{\prime}$ for $x>0$. The asymptotic wave functions in terms of $k$ and $k^{\prime}$ are thus given by

$$
\begin{aligned}
\lim _{x \rightarrow-\infty} \psi(x) & \simeq e^{i k^{\prime} x}+r_{\text {left }}\left(k, k^{\prime}\right) e^{-i k x} \\
\text { and } \quad \lim _{x \rightarrow+\infty} \psi(x) & \simeq t_{l e f t}\left(k, k^{\prime}\right) e^{i k^{\prime} x}
\end{aligned}
$$

where $r_{\text {left }}\left(k, k^{\prime}\right)$ and $t_{\text {left }}\left(k, k^{\prime}\right)$ are reflection and transmission amplitudes due to left incident wave. Similarly for the right incident wave we have

$$
\begin{aligned}
\lim _{x \rightarrow \infty} \psi(x) & \simeq e^{-i k x}+r_{\text {right }}\left(k, k^{\prime}\right) e^{i k^{\prime} x} \\
\text { and } \quad \lim _{x \rightarrow-\infty} \psi(x) & \simeq t_{\text {right }}\left(k, k^{\prime}\right) e^{-i k x} .
\end{aligned}
$$


The reflectivity $R\left(k, k^{\prime}\right)$ and the transmitivity $T\left(k, k^{\prime}\right)$ are

$$
R\left(k, k^{\prime}\right)=\left|r\left(k, k^{\prime}\right)\right|^{2} \quad \text { and } \quad T\left(k, k^{\prime}\right)=\left|t\left(k, k^{\prime}\right)\right|^{2}
$$

respectively.

For this asymptotically non-vanishing potential, the bound state energy eigenvalues and the eigenfunctions are given by Eqs. (18) and (20) respectively. For $B>0$, the non-vanishing part of the potential will be emissive for $x>0$ and absorptive for $x<0$. Due to this reason the asymptotic wave numbers $k$ and $k^{\prime}$ will be complex and expressed in terms of $\alpha_{n}$ and $\beta_{n}$ as

$$
\text { and } \quad \begin{aligned}
& E_{n}-V^{(-)}(x \rightarrow+\infty)=E_{n}-2 i B=-\alpha_{n}^{2}=k^{2} \\
& E_{n}-V^{(-)}(x \rightarrow-\infty)=E_{n}+2 i B=-\beta_{n}^{2}=k^{\prime 2} .
\end{aligned}
$$

Thus the complex parameters $\alpha_{n}$ and $\beta_{n}$ can be written as

$$
\alpha_{n}=i k^{\prime} \quad \beta_{n}=i k .
$$

Eq. (19) when expressed in terms of hypergeometric function, takes the form

$$
\psi_{n}^{(+)}(x) \propto(1-\tanh x)^{\frac{\alpha_{n}}{2}}(1+\tanh x)^{\frac{\beta_{n}}{2}} F\left(n+\alpha_{n}+\beta_{n}+1,-n ; 1+\alpha_{n} ; \frac{1-\tanh x}{2}\right) .
$$

Now we also have to consider the second solution of the hypergeometric function by using Eq. (32). The wavefunction $\psi_{n}^{(+)}(x)$ at $x \longrightarrow \infty$ takes the form

$$
\lim _{x \rightarrow \infty} \psi_{n}^{(+)}(x) \propto\left(C_{1} e^{-\alpha_{n} x}+C_{2} e^{\alpha_{n} x}\right) .
$$

To get the asymptotic solutions at $x \rightarrow-\infty$ we use another property of the hypergeometric functions 42

$$
\begin{aligned}
F(a, b ; c ; z)= & \frac{\Gamma(c) \Gamma(c-a-b)}{\Gamma(c-a) \Gamma(c-b)} F(a, b ; a+b-c+1 ; 1-z)+(1-z)^{c-a-b} \\
& \frac{\Gamma(c) \Gamma(a+b-c)}{\Gamma(a) \Gamma(b)} F(c-a, c-b ; c-a-b+1 ; 1-z)
\end{aligned}
$$

in Eq. (54) and get

$$
\lim _{x \rightarrow-\infty} \psi_{n}^{(+)}(x) \propto\left(\left(C_{1} \chi_{1}+C_{2} \chi_{2}\right) e^{\beta_{n} x}+\left(C_{1} \chi_{3}+C_{2} \chi_{4}\right) e^{-\beta_{n} x}\right),
$$

where

$$
\begin{array}{ll}
\chi_{1}=\frac{\Gamma\left(1+\alpha_{\nu}\right) \Gamma\left(-\beta_{\nu}\right)}{\Gamma\left(-\nu-\beta_{\nu}\right) \Gamma\left(1+\alpha_{\nu}+\nu\right)} ; & \chi_{3}=\frac{\Gamma\left(1+\alpha_{\nu}\right) \Gamma\left(\beta_{\nu}\right)}{\Gamma\left(\nu+\alpha_{\nu}+\beta_{\nu}+1\right) \Gamma(-\nu)} \\
\chi_{2}=\frac{\Gamma\left(1-\alpha_{\nu}\right) \Gamma\left(-\beta_{\nu}\right)}{\Gamma\left(-\alpha_{\nu}-\beta_{\nu}-\nu\right) \Gamma(1+\nu)} ; & \chi_{4}=\frac{\left.\Gamma\left(1-\alpha_{\nu}\right) \Gamma\left(\beta_{\nu}\right)\right)}{\Gamma\left(\nu+\beta_{\nu}+1\right) \Gamma\left(-\nu-\alpha_{\nu}\right)} .
\end{array}
$$


Using Eq. (19) the asymptotic wavefunctions of the rationally extended complex RosenMorse II potential are given by

$$
\begin{aligned}
\lim _{x \rightarrow \infty} \psi_{\nu}^{-}(x) & \propto \lim _{x \rightarrow \infty}\left[\hat{A} \psi_{\nu}^{+}(x)\right] \\
& =C_{1}\left(\alpha_{m}-i k^{\prime}\right) e^{-i k^{\prime} x}+C_{2}\left(\alpha_{m}+i k^{\prime}\right) e^{i k^{\prime} x}
\end{aligned}
$$

and

$$
\begin{aligned}
\lim _{x \rightarrow-\infty} \psi_{\nu}^{-}(x) & \propto \lim _{x \rightarrow-\infty}\left[\hat{A} \psi_{\nu}^{+}(x)\right] \\
& =\left[\left(C_{1} \chi_{1}+C_{2} \chi_{2}\right)\left(i k-\beta_{m}\right)\right] e^{i k x}+\left[\left(C_{1} \chi_{3}+C_{2} \chi_{4}\right)\left(-i k-\beta_{m}\right)\right] e^{-i k x}
\end{aligned}
$$

Now comparing Eq. (49) with Eq. (59), we get the constants

$$
C_{1}=0 ; \quad \text { and } \quad C_{2}=\frac{1}{\chi_{2}\left(i k-\beta_{m}\right)},
$$

with the same $\alpha_{m}$ and $\beta_{m}$ given in Eq. (17).

Using $\chi_{2}$ and the above constants $C_{1}$ and $C_{2}$, we can easily obtain the scattering amplitudes due to left incident wave

$$
t_{l e f t}\left(k, k^{\prime}, m\right)=\frac{\left(\alpha_{m}+i k^{\prime}\right)}{\left(i k-\beta_{m}\right)} \times t_{\text {left }}^{u s u a l}\left(k, k^{\prime}\right)
$$

and

$$
r_{\text {left }}\left(k, k^{\prime}, m\right)=\frac{\left(-\beta_{m}-i k\right)}{\left(-\beta_{m}+i k\right)} \times r_{\text {left }}^{\text {usual }}\left(k, k^{\prime}\right),
$$

where $t_{\text {left }}^{\text {usual }}\left(k, k^{\prime}\right)$ and $r_{\text {left }}^{\text {usual }}\left(k, k^{\prime}\right)$ are the left transmission and reflection amplitudes for the usual PT symmetric complex Rosen-Morse potential [41] given by

$$
t_{\text {left }}^{\text {usual }}\left(k, k^{\prime}\right)=\frac{\Gamma\left(-A-1-\frac{i k^{\prime}}{2}-\frac{i k}{2}\right) \Gamma\left(A+2-\frac{i k^{\prime}}{2}-\frac{i k}{2}\right)}{\Gamma\left(1-i k^{\prime}\right) \Gamma(-i k)}
$$

and

$$
r_{\text {left }}^{\text {usual }}\left(k, k^{\prime}\right)=\frac{\Gamma(i k)}{\Gamma(-i k)} \frac{\Gamma\left(-A-1-\frac{i k^{\prime}}{2}-\frac{i k}{2}\right) \Gamma\left(A+2-\frac{i k^{\prime}}{2}-\frac{i k}{2}\right)}{\Gamma\left(-A-1-\frac{i k^{\prime}}{2}+\frac{i k}{2}\right) \Gamma\left(A+2-\frac{i k^{\prime}}{2}+\frac{i k}{2}\right)} .
$$

Similarly from Eqs. (50) and (59) we can easily obtain

$$
\begin{aligned}
t_{\text {right }}\left(k, k^{\prime}, m\right) & =\frac{\left(-\beta_{m}-i k\right)}{\left(\alpha_{m}-i k^{\prime}\right)}\left(\frac{k^{\prime}}{k}\right) \frac{\Gamma\left(-A-1-\frac{i k^{\prime}}{2}-\frac{i k}{2}\right) \Gamma\left(A+2-\frac{i k^{\prime}}{2}-\frac{i k}{2}\right)}{\Gamma\left(1-i k^{\prime}\right) \Gamma(-i k)} \\
& =\left(\frac{\beta_{m}-i k}{\alpha_{m}-i k^{\prime}}\right)\left(\frac{i k-\beta_{m}}{i k^{\prime}+\alpha_{m}}\right)\left(\frac{k^{\prime}}{k}\right) t_{\text {left }}\left(k, k^{\prime}, m\right)
\end{aligned}
$$


and

$$
r_{\text {right }}\left(k, k^{\prime}, m\right)=\frac{\left(\alpha_{m}+i k^{\prime}\right)}{\left(\alpha_{m}-i k^{\prime}\right)} \frac{\Gamma\left(i k^{\prime}\right)}{\Gamma\left(-i k^{\prime}\right)} \frac{\Gamma\left(-A-1-\frac{i k^{\prime}}{2}-\frac{i k}{2}\right) \Gamma\left(A+2-\frac{i k^{\prime}}{2}-\frac{i k}{2}\right)}{\Gamma\left(-A-1+\frac{i k^{\prime}}{2}-\frac{i k}{2}\right) \Gamma\left(A+2+\frac{i k^{\prime}}{2}-\frac{i k}{2}\right)} .
$$

\section{Remarks:}

- Similar to the extended PT invariant complex Scarf II case, the left/right scattering amplitudes for the extended complex Rosen-Morse II potential are also modified with an extra $m$ dependent factor. As expected, in the special case of $m=0$ these results match exactly with those of complex PT invariant RM-II.

- Due to the asymptotically non-vanishing behavior of the extended Rosen-Morse II potential, from Eqs. (61) and (64) we observe that on changing the direction of the incoming wave, the transmission amplitude too changes by a phase factor

of $\left(\frac{\beta_{m}-i k}{\alpha_{m}-i k^{\prime}}\right)\left(\frac{i k-\beta_{m}}{i k^{\prime}+\alpha_{m}}\right)\left(\frac{k^{\prime}}{k}\right)$. Further, as in the extended complex Scarf II case, the reflection amplitude for this potential also exhibits the handedness effect.

- Since the absolute value of the extra term is one, hence the scattering properties such as the unitarity and the reciprocity will be the same for the complex extended $P T$ invariant as well as the conventional complex PT invariant Rosen-Morse II potential

\section{Summary and Conclusions}

In this paper, we have filled the missing gap by discussing the bound state eigenfunctions of the rationally extended PT symmetric complex Scarf II and Rosen-Morse II potentials. The eigenvalues of these potentials are isospectral to their conventional counterparts and the eigenfunctions are written in terms of $X_{m}$ EOPs and some new types of polynomials respectively. Further, we have briefly discussed the parametric symmetry related to the extended Scarf II potential. The reflection and the transmission amplitudes have also been calculated for these potentials and we have shown that as we go from the case of the conventional to the extended case, we get the modified scattering amplitudes which are now $m$ dependent. Some of the important scattering properties related to these potentials such as the handedness effect, the reciprocity and the unitarity have also been discussed.

\section{Acknowledgments}

BPM acknowledges the financial support from the Department of Science and Technology (DST), Govt. of India under SERC project sanction grant No. SR/S2/HEP0009/2012. AK would like to thank Indian National Science Academy (INSA) for the award of INSA senior scientist position at Savitribai Phule Pune University, Pune. 


\section{References}

[1] D. Gomez-Ullate, N. Kamran and R. Milson, J. Math. Anal.Appl. 359 (2009) 352.

[2] D. Gomez-Ullate, N. Kamran and R. Milson, J. Phys. A 43 (2010) 434016.

[3] D. Gomez-Ullate, Y. Grandati and R. Milson, J. Phys. A 47 (2014) 015203.

[4] D. Gomez-Ullate, N. Kamran and R. Milson, Contemporary Mathematics 56351 2012.

[5] C. Quesne, J.Phys.A 41 (2008) 392001.

[6] B. Bagchi, C. Quesne and R. Roychoudhary, Pramana J. Phys. 73(2009) 337.

[7] S. Odake and R. Sasaki, Phys. Lett. B, 684 (2010) 173; ibid 679 (2009) 414. J. Math. Phys, 51, 053513 (2010).

[8] R. K. Yadav, N. Kumari, A. Khare and B. P. Mandal, arXiv: 1412.5445 [quant-ph].

[9] F. Cooper, A. Khare, U. Sukhatme, Phys. Rep. 251 (1995) 267; "SUSY in Quantum Mechanics" World Scientific (2001).

[10] C. Quesne, SIGMA 8 (2012) 080.

[11] C. Quesne, I. J. Mod. Phys. A 27 (2012) 1250073.

[12] C. M. Bender and S. Boettcher, Phys. Rev. Lett. 80 (1998) 5243.

C. M. Bender, Rep. Prog. Phys. 70 (2007) 947 and references therein.

[13] A. Mostafazadeh, J. Math. Phys., 43 (2002) 205; ibid 43 (2002) 2814.

[14] Z. H. Musslimani, K. G. Makris, R. El-Ganainy and D. N. Christodulides Phys. Rev. Lett. 100 (2008) 030402.

[15] C. E. Rutter et.al., Nature Phys. 6 (2010) 192.

[16] K. G. Makris, R. El-Ganainy, D. N. Christodulides and Z. H. Musslimani, Phys. Rev. Lett. 100 (2008) 103904.

[17] A. K. Pati, Pramana J. Phys. 733 (2009) 485.

[18] C. M. Bender et.al., Phys. Rev. Lett. 98 (2007) 040403.

[19] A. Khare and B. P. Mandal,Phys. Lett. A 53 (2000) 272.

[20] B. P. Mandal, B. K. Mourya and R. K. Yadav, Phys. Lett. A 377 (2013) 1043.

[21] B. B. Mallick, T. Bhattacharya and B. P. Mandal, Modern Phys. Lett. A 20 (2004) 543; B. B. Mallick and B. P. Mandal, Phys. Lett. A 284 (2001) 231. 
[22] B.F. Samsonov, J. Phys. A 38 (2005) L571.

[23] A. Mostafazadeh, J. Phys. A 44 (2011) 375302.

[24] Z. Ahmed, J. Phys. A 42 (2009) 472005.

[25] A. Mostafazadeh and M. Sarisman, Phys. Lett. A 375 (2011) 3387.

[26] A. Ghatak, R. D. R. Mandal and B. P. Mandal, Annals of Physics 336 (2013) 540.

[27] W.D. Heiss, J. Phys. A 37 (2004) 2455.

[28] M.V. Berry, Czech. J. Phys. 54 (2004) 1039.

[29] A.V. Smilga, J. Phys. A 42 (2009) 095301.

[30] M. Muller and I. Rotter, J. Phys. A 41 (2008) 244018.

[31] Z. Ahmed, Phys. Lett. A 324 (2004) 152; ibid 377 (2013) 957; J. Phys. A45 (2012) 032004 .

[32] B. Bagchi, C. Quesne, J. Phys. A: Math. Theor. 43 (2010) 305301.

[33] B. Midya and B. Roy, J. Phys. A 46 (2013) 175201.

[34] R. K. Yadav, A. Khare and B. P. Mandal, Annals of Physics 331 (2013) 313.

[35] R. K. Yadav, A. Khare and B. P. Mandal, Phys. Lett. B 723 (2013) 433.

[36] R. K. Yadav, A. Khare and B. P. Mandal, Phys. Lett. A 379 (2015) 67.

[37] C. L. Ho, J. C. Lee and R. Sasaki, Annals of Physics 343 (2014) 115.

[38] R. K. Yadav, A. Khare, N. Kumari, B. Bagchi and B. P. Mandal, arXiv: 1510.01666 [quant-ph].

[39] B. Bagchi and C. Quesne, Phys. Lett. A 300 (2002) 18.

[40] B. Bagchi and C. Quesne, Phys. Lett. A 273 (2000) 285.

[41] G. Levai and E. Magyari, J. Phys. A: Math. Theor. 42 (2009) 195302.

[42] I. S. Gradshteyn, I. M. Ryzhik, Alan Jeffrey, "Table of Integral, Series and Product", Academic Press (1991).

[43] Z. Ahmed, Phys. Lett. A 282 (2001) 343; 287 (2001) 295.

[44] Z. Ahmed, Phys. Lett. A 377 (2013) 957. 